\title{
Reapertura de laboratorios de motilidad durante la pandemia de COVID-19, regurgitación o rumiación, neurogastro en la práctica clínica y disbiosis en niños con TIIC
}

Miguel Saps ${ }^{1}$ y Max Schmulson ${ }^{2 *}$

${ }^{1}$ Department of Pediatrics, Division of Pediatric Gastroenterology, Hepatology and Nutrition, University of Miami-Miller School of Medicine, Miami, Fla.;

${ }^{2}$ Profesor Titular de Medicina, Laboratorio de Hígado, Páncreas y Motilidad (HIPAM)-Unidad de Investigación en Medicina Experimental, Facultad de

Medicina-Universidad Nacional Autónoma de México, Ciudad de México, México

La actual pandemia de enfermedad por coronavirus 2019 (COVID-19) ha limitado la práctica de la medicina en especialidades no directamente relacionadas con la infectología, la neumología o la terapia intensiva, tales como la gastroenterología y nuestro propio campo, la neurogastroenterología. Es así como actualmente existen guías internacionales para la reapertura de unidades de endoscopia y laboratorios de fisiología y/o motilidad gastrointestinal y uso de equipo de protección personal (EPP) que prevenga la diseminación de la infección por COVID-19 a nivel hospitalario y hacia el personal de salud ${ }^{1-4}$. Pero la pandemia no solo ha impactado el ejercicio de la medicina, sino consecuentemente y de manera muy negativa en el ingreso económico de los

Correspondence to:

*Max Schmulson-Wasserman

Laboratorio de Hígado, Páncreas y Motilidad (HIPAM)

Unidad de Investigación en Medicina Experimental

Facultad de Medicina-Universidad Nacional Autónoma de México

(UNAM)

Dr. Balmis, 148

Col. Doctores

C.P. 06726, Ciudad de México, México

E-mail:maxjulio@prodigy.net.mx médicos, que en países como los EE.UU. han requerido programas de ayuda financiera ${ }^{5}$. De hecho en un estudio que llevamos a cabo entre miembros de la Sociedad Latinoamericana de Neurogastroenterología (SLNG) encontramos que el $100 \%$ de los que respondieron la encuesta correspondiente reportó una reducción de su práctica y, de manera preocupante, el $88.6 \%$ informó una disminución del 61 al 100\%; lo que sugiere que un grupo de neurogastroenterólogos de nuestra Sociedad quedaron sin fuente de ingresos ${ }^{6}$. Además, si bien el $83 \%$ implementó la telemedicina, solo el $64.7 \%$ de ellos eran remunerados por esta práctica. Pero preocupante también fue el hallazgo de que a pesar de no existir al momento de la encuesta guías latinoamericanas para el reinicio de
Received in original form: 20-08-2020

Accepted in final form: 26-08-2020

DOI: 10.24875/NGL.M20000017 
actividades de laboratorios de fisiología/motilidad, y quizá las recomendaciones internacionales eran en ese momento muy escuetas, el 96\% reportó continuar con estos procedimientos $^{6}$.

Posterior a esta encuesta, la SLNG publicó guías para la reapertura y reinicio de los laboratorios de motilidad debido a que estos procedimientos implican un riesgo intermedio o elevado de transmisión de la COVID-197. Sin embargo, la región latinoamericana es muy variable en cuanto a la tasa de infección, por lo cual la SLNG ha recomendado diferir estos procedimientos diagnósticos y terapéuticos hasta que se logre un control de la infección en cada país. Estas guías han considerado de alto riesgo procedimientos como manometría esofágica, pHmetría-impedancia, pH-metría con cápsula inalámbrica, manometría antroduodenal y pruebas de aliento, debido a la posibilidad de aerosolización del coronavirus 2 del síndrome respiratorio agudo grave (SARS-CoV-2) ${ }^{7}$. Dichas guías (SLNG) han clasificado en cambio como riesgo intermedio a la manometría anorrectal, biorretroalimentación, prueba de expulsión con balón, electromiografía, manometría colónica y pruebas con barostato como de riesgo intermedio, por lo cual deben ser diferidos7. Lo anterior debido a que existen datos en la literatura de excreción de partículas virales en heces ${ }^{8-11}$. Así mismo, el tránsito colónico con marcadores radioopacos o la cápsula inteligente fueron considerados de riesgo intermedio, por la exposición del personal de salud a pacientes que potencialmente pudieran ser vectores de la COVID-197. Es por ello que Viebig y GranjaAndrade han revisado en este número de NGLR las recomendaciones para realizar procedimientos de manometría con base en los lineamientos internacionales pero adaptados a la región latinoamericana ${ }^{12}$. Los autores recomiendan que cuando los laboratorios de motilidad puedan regresar a su labor normal con base en las políticas locales de salud y los datos epidemiológicos de cada país o región, se programe inicialmente los procedimientos de mayor urgencia, seguidos de los electivos. Viebig y Granja-Andrade mencionan la utilidad de recomendar pruebas de reacción en cadena de la polimerasa (PCR) para el SARSCoV-2 o incluso pruebas rápidas antes del procedimiento, lo cual puede no ser factible en la realidad económica latinoamericana. Sin embargo, mientras no tengamos vacunas ni tratamiento para la COVID-19, es muy probable que tengamos que proseguir con los procedimientos de motilidad esofágica y anorrectal en los diversos laboratorios, haciendo uso del EPP, y ante todo, un cuestionario con triaje para determinar factores de riesgo de los pacientes candidatos ${ }^{12}$. Si bien las pruebas diagnósticas como la PCR realizada de 48 a 72 horas antes del procedimiento pueden incrementar los costos, a juicio de los editores abajo firmantes, debe ser realizada en la medida de lo posible. Viebig y Granja-Andrade proponen que si la PCR es positiva o existe alta sospecha de que el paciente candidato se encuentre infectado se deben posponer los procedimientos de seis a ocho semanas; o si el paciente estuvo en contacto con un caso positivo, entonces posponer 14 días hasta determinar si no desarrolló COVID-1912. Recomiendan además aplicar un nuevo cuestionario de triaje inmediatamente antes de iniciar el procedimiento. Finalmente, Viebig y Granja-Andrade también describen las medidas de desinfección del laboratorio y de todos los elementos del equipo posterior a la realización de los estudios diagnósticos y terapéuticos en estas 
unidades, lo cual será de utilidad para nuestros lectores ${ }^{12}$.

El segundo artículo de este número, firmado por García, et al., aborda un par de problemas frecuentes en la práctica clínica que resultan en un dilema de diagnóstico diferencial pero sobre todo de tratamiento: regurgitación vs. rumiación ${ }^{13}$. Los autores pertenecen al grupo del Dr. Daniel Sifrim, autor sénior de esta revisión y orgullosamente un argentino que trabaja en el Barts and the London School of Medicine and Dentistry y el Royal London Hospital, líderes internacionales en el tema ${ }^{14-16}$. Regurgitación definida como la percepción del retorno repentino y sin esfuerzo del contenido gástrico hacia la boca o la hipofaringe en contraste con rumiación, caracterizada por la regurgitación a la boca de la comida recientemente ingerida en ausencia de anormalidades estructurales ${ }^{13}$. El primero, un síntoma que puede ser secundario a trastornos como el reflujo gastroesofágico, pero con menor respuesta a los inhibidores de la bomba de protones (IBP), que también puede estar presente en la acalasia o gastroparesia ${ }^{17,18}$. En contraste, la rumiación es un trastorno gastroduodenal, dentro de los trastornos de la interacción intestino-cerebro (TIIC), que se clasifica mediante los Criterios de Roma IV ${ }^{19}$. El recién publicado Estudio Epidemiológico Global de Roma IV encontró una prevalencia de rumiación del 2.8\% (intervalo de confianza del $95 \%$ : 2.7-2.9) entre 54,127 sujetos de 26 países encuestados por Internet y 18,949 sujetos de nueve países encuestados cara a cara, además de presentar mayor frecuencia en el grupo de 40 a 64 años de edad ${ }^{20}$. Hay que resaltar que en este estudio global de Roma IV se incluyeron cuatro países latinoamericanos (México, Colombia, Argentina y Brasil), todos encuestados por Internet ${ }^{20}$, sin embargo, la prevalencia de Rumiación de cada uno de los países aún no ha sido analizada.

García, et al. destacan la utilidad de métodos diagnósticos como la monitorización combinada de impedancia intraluminal multicanal y $\mathrm{pH}$, resaltando que este método ha permitido conocer que los episodios de reflujo gastroesofágico (RGE) que inducen regurgitación, se caracterizan por una extensión más proximal que los episodios de RGE que provocan pirosis. Debido a su extensión más proximal, desencadenan además otros síntomas extraesofágicos que comprometen la faringe, cavidad oral, nariz, senos paranasales y hasta el oído medio ${ }^{13}$. En cuanto al tratamiento, refieren los autores que si bien los pacientes con regurgitación responden menos a los IBP, la funduplicatura es una buena posibilidad terapéutica en los pacientes que sí responden a estos agentes, en especial si el paciente tiene hernia hiatal. Por otra parte, en los pacientes que no responden a IBP y sin hernia hiatal, la primera opción es funduplicatura transoral sin incisión (TIF), seguida del aumento esfinteriano magnético (MSA) y funduplicatura laparoscópica, y en aquellos con hernia hiatal, la primera opción es funduplicatura o MSA ${ }^{21}$. En cambio, en los pacientes con respuesta parcial o total a IBP con hernia hiatal, funduplicatura o MSA, mientras que, en aquellos sin hernia, se puede considerar funduplicatura laparoscópica o MSA, seguidas de TIF $^{21}$. En cuanto a la rumiación, a pesar del diagnóstico con los criterios de Roma IV, destacan también la confirmación objetiva mediante manometría esofágica de alta resolución combinada con impedanciometría ${ }^{14}$, tecnología que no está disponible universalmente, por lo cual la clínica es de suma importancia. Por ejemplo, a 
diferencia del vómito, la rumiación no está precedida de náuseas ${ }^{22}$. Es importante recalcar que la rumiación se ha caracterizado como un trastorno de la conducta y como una consecuencia al estrés ${ }^{22-24}$, incluso con criterios de DSM-5 de los Desórdenes mentales:« Rumiación primaria» ${ }^{25}$. Como tal, el tratamiento es complejo y en estos casos la terapia cognitiva conductual es la primera opción ${ }^{26,27}$. En cambio en rumiación secundaria por RGE, tratamientos dirigidos como el baclofeno y la funduplicatura son de utilidad $^{28}$.

El tercer artículo de este número revisa la importancia de la neurogastroenterología en la práctica diaria del gastroenterólogo general ${ }^{29}$. El artículo analiza y compara los trastornos motores y los TIIC, desde la deglución a la evacuación, conceptualizando los desórdenes con un criterio fisiopatológico práctico en desórdenes de la deglución, enfermedad por RGE, alteraciones del vaciamiento gástrico, trastornos de la digestión, motilidad intestinal y colónica, y desórdenes de la función anorrectal $^{29}$. Asimismo, detalla la serie de estudios que permiten al gastroenterólogo refinar su diagnóstico y consecuentemente el tratamiento. Estos estudios incluyen la manometría de alta resolución esofágica y anorrectal, estudios de vaciamiento gástrico por medicina nuclear, defecografía por resonancia magnética y pruebas de aliento para sobrepoblación bacteriana del intestino delgado ${ }^{30-34}$. Continuando con un aspecto práctico que permite al gastroenterólogo diagnosticar a los pacientes en forma clínica, se enlistan los trastornos según los criterios de Roma IV y basados en el órgano blanco que comprometen. Un diagnóstico clínico es de especial importancia ante la ausencia de marcadores biológicos establecidos para diagnosticar y diferenciar los distintos TIIC.
Los criterios de Roma, de esta forma y mediante la estandarización diagnóstica, también permiten una mejor comunicación entre los médicos, facilitando la interconsulta $y$ transición entre especialistas, y ayuda a evitar la repetición de estudios y la inclusión de los pacientes en protocolos de investigación. El artículo ofrece una tabla de suma practicidad, incluyendo los trastornos de la motilidad y los trastornos de la TIIC según el órgano blanco afectado. Esto demuestra la estrecha relación entre ambos grupos de trastornos con aplicación práctica para el gastroenterólogo en la clínica diaria. El artículo luego revisa algunos de los trastornos de la motilidad y TIIC más comunes, su manejo clínico y los estudios que ayudan en su diagnóstico y diferenciación clínica, incluyendo disfagia, enfermedad por RGE, dispepsia funcional y gastroparesia, trastornos de la defecación y el síndrome del intestino narcótico. Este último, también conocido como hiperalgesia gastrointestinal inducida por opioides, fue introducido en Roma $I^{35}$ y se caracteriza por el empeoramiento del dolor abdominal secundario al uso de dosis continuas o progresivamente mayores de opioides y debe ser diferenciado del trastorno de la defecación inducido por opioides, que resulta del accionar de los opioides sobre la motilidad y secreción gastrointestinal $^{36}$. El artículo incluye el algoritmo de Roma IV que es muy práctico para el diagnóstico del síndrome del intestino narcótico en pacientes que reciben altas dosis de narcóticos o son tratados crónicamente con este tipo de fármacos para el manejo del dolor abdominal ${ }^{29}$. Esta exhaustiva revisión también proporciona una descripción del perfil clínico multidimensional en los $\mathrm{TIIC}^{37}$ y los requisitos de conocimiento para gastroenterólogos en diferentes niveles de entrenamiento, que han sido 
delineados por la American Neurogastroenterology and Motility Society y que incluyen la transición del paciente pediátrico al gastroenterólogo de adultos y expectativas para el futuro ${ }^{38}$.

El último artículo, de Jacquez, et al., revisa la posible relación entre microbiota y obesidad en niños con trastornos gastrointestinales funcionales $^{39}$. Si bien muchos artículos han investigado la relación entre obesidad y trastornos gastrointestinales en niños, no ha habido revisiones para determinar si los cambios en la microbiota podrían constituir el enlace entre estas dos patologías. En esta revisión, los autores describen la relación entre los diversos TIIC pediátricos y obesidad, la función de la microbiota en general y los cambios de la microbiota en cada una de estas patologías. Como ejemplo, el artículo cita cambios en la composición de la microbiota en niños con síndrome de intestino irritable (SII) postinfección ${ }^{40}$. En ellos mediante el estudio de 16S, metagenómico, con PhyloChip de hibridación de DNA y pirosecuenciación 454, encontraron que la mayor frecuencia de dolor abdominal estaba asociada con un aumento de taxas bacterianas del género Alistipes ${ }^{40}$. Así mismo, el artículo cubre el uso de probióticos incluyendo Lactobacillus rhamnosus GG, para el cual ha habido múltiples estudios aleatorizados además de un metaanálisis que ha mostrando su eficacia para el tratamiento del dolor abdominal funcional y SII en particular en niños, con un número necesario para tratar de 4 y 7 respectivamente $^{41}$. La revisión también cita el estudio de Zoppi, et al. sobre estreñimiento en niños, en el cual se encontró que este grupo de pacientes tenía un incremento de Clostridia y Bifidobacterium en heces, comparados con los controles ${ }^{42}$. Los autores también revisan la relación de la obesidad con cambios en la microbiota. No solo revisan la relación de la microbiota con la obesidad en niños, sino también varios artículos sobre la microbiota infantil en general y luego sobre la obesidad en particular ${ }^{39}$. Es de destacar el estudio de Bergström, et al., donde se analiza el desarrollo de la microbiota en lactantes. En ese estudio los autores encuentran que aproximadamente a los 9 meses de vida, cuando se establecen cambios importantes en la alimentación, se produce un aumento de Lactobacilli, Bifidobacterium y Enterobacteriaceae, con una microbiota dominada por Clostridium spp. y Bacteroides spp. Con respecto a la obesidad ${ }^{43}$, Million, et al. encontraron en modelos animales una asociación de Firmicutes con obesidad y del filotipo Bacteroidetes con pérdida de peso $^{44}$. Estudios en adolescentes mostraron que la reducción en la proporción de Clostridium histolyticum y Eubacterium rectale - Clostridium coccoides se correlacionó con una reducción en el índice de masa corporal y peso ${ }^{45}$. Lamentablemente la revisión no encontró artículos que hubieran estudiado la microbiota en niños con los TIIC con obesidad en comparación con controles, indicando un área de posible desarrollo e investigación.

Así pues, este es un número más de NGLR en medio de la pandemia de COVID-19 que no ha dejado de afectar nuestra práctica, pero que debemos considerar en el reinicio de las actividades de los laboratorios de motilidad y/o fisiología. Lo anterior como parte de la neurogastroenterología, hoy, por hoy, quizá el área más extensa dentro de la gastroenterología clínica. Regurgitación vs. rumiación es un claro ejemplo de los problemas a los que nos enfrentamos dentro de nuestra práctica clínica. Finalmente, el efecto de la microbiota como mediador de TIIC y obesidad, y 


\section{campo no solo de actualidad en la práctica pediátrica, sino también de los adultos.}

\section{BIBLIOGRAFÍA}

1. Onoyama T, Isomoto H. COVID-19 and gastrointestinal endoscopy: Importance of reducing SARS-CoV-2 infection risks of medical workers and preserving personal protective equipment resources. Dig Endosc. 2020 May 13;10.1111/den.13720. doi: 10.1111/den.13720. Online ahead of print.

2. Gastroenterology Professional Society Guidance on Endoscopic Procedures during the COVID-19 Pandemic [Internet]. American College of Gastroenterology [fecha de publicación: 1 de abril de 2020]. Disponible en: https://gi. org/2020/04/01/joint-gi-society-message-on-endoscopy-during-covid-19/

3. Sociedad Española de Patología Digestiva; Asociación Española de Gastroenterología. Recommendations by the SEPD and AEG, both in general and on the operation of gastrointestinal endoscopy and gastroenterology units, concerning the current SARS-CoV-2 pandemic (March, 18). Rev Esp Enferm Dig. 2020;112(4):319-22.

4. Baker JR, Moshiree B, Rao S, Neshatian L, Nguyen L, Chey WD, et al. American Neurogastroenterology and Motility Society Task Force Recommendations for Resumption of Motility Laboratory Operations During the COVID-19 Pandemic. Am J Gastroenterol. 2020 Aug 28. doi: 10.14309/ ajg.0000000000000823. Online ahead of print.

5. Guide to COVID-19 financial assistance programs for physicians [Internet]. American Gastroenterological Association [fecha de publicación: 31 de marzo de 2020. Disponible en: https://aga-cms-assets.s3.amazonaws. com/202033120334---ADV20-026\%20COVID-19\%20Financial\%20Assistance $\% 20$ Programs_FINAL.pdf

6. Schmulson M, Gudiño-Zayas M; Sociedad Latinoamericana de Neurogastroenterología. The impact of COVID-19 pandemic on neurogastroenterologists in Latin America: Results of an online survey. J Clin Gastroenterol. 2020: Advancen on-line publication.

7. Remes-Troche JM, Valdovinos-Diaz MA, Viebig R, Defilippi C, Bustos-Fernández LM, Sole L, et al. Recommendations for the reopening and activity resumption of the neurogastroenterology units in the face of the COVID-19 pandemic. Position of the Sociedad Latinoamericana de Neurogastroenterologia. Rev Gastroenterol Mex. 2020 Jul 11;S0375-0906(20)300835. doi: 10.1016/j.rgmx.2020.07.001. Online ahead of print.

8. Park SK, Lee CW, Park DI, Woo HY, Cheong HS, Shin HC, et al. Detection of SARS-CoV-2 in fecal samples from patients with asymptomatic and mild COVID-19 in Korea. Clin Gastroenterol Hepatol. 2020 Jun 10;S15423565(20)30777-1. doi: 10.1016/j.cgh.2020.06.005. Online ahead of print.

9. Yeo C, Kaushal S, Yeo D. Enteric involvement of coronaviruses: is faecal-oral transmission of SARS-CoV-2 possible? Lancet Gastroenterol Hepatol. 2020;5:335-7.

10. Zhang J, Wang S, Xue Y. Fecal specimen diagnosis 2019 novel coronavirus-infected pneumonia. J Med Virol. 2020;92(6):680-2.

11. Wu Y, Guo C, Tang L, Hong Z, Zhou J, Dong X, et al. Prolonged presence of SARS-CoV-2 viral RNA in faecal samples. Lancet Gastroenterol Hepatol. 2020;5:434-5

12. Viebig RG, Granja-Andrade CG. Should we perform esophageal and/or anorectal manometries during the COVID-19 pandemic? Neurogastro LATAM Rev. 2020;4:68-75.

13. García K, Guzmán M, Souza M, Sifrim D. Regurgitación vs. rumiación: diagnóstico y tratamiento. Neurogastro LATAM Rev. 2020;4:95-109.

14. Nakagawa K, Sawada A, Hoshikawa Y, Nikaki K, Sonmez S, Woodland P, et al. Persistent postprandial regurgitation vs rumination in patients with refractory gastroesophageal reflux disease symptoms: Identification of a distinct rumination pattern using ambulatory impedance-pH monitoring. Am J Gastroenterol. 2019;114:1248-55.
15. Wang YT, Tai LF, Yazaki E, Jafari J, Sweis R, Tucker E, et al. Investigation of dysphagia after antireflux surgery by high-resolution manometry: Impact of multiple water swallows and a solid test meal on diagnosis, management, and clinical outcome. Clin Gastroenterol Hepatol. 2015;13:1575-83.

16. Rommel N, Tack J, Arts J, Caenepeel P, Bisschops R, Sifrim D. Rumination or belching-regurgitation? Differential diagnosis using oesophageal impedance-manometry. Neurogastroenterol Motil. 2010;22:e97-104

17. El-Serag HB, Sweet S, Winchester CC, Dent J. Update on the epidemiology of gastro-oesophageal reflux disease: a systematic review. Gut. 2014;63:871-80.

18. Cappell MS, Stavropoulos SN. Updated systematic review of achalasia, with a focus on POEM therapy. 2020;65:38-65.

19. Stanghellini V, Chan FK, Hasler WL, Malagelada JR, Suzuki H, Tack J, et al. Gastroduodenal disorders. Gastroenterology. 2016;150:1380-92.

20. Sperber AD, Bangdiwala SI, Drossman DA, Ghoshal UC, Simren M, Tack J, et al. Worldwide prevalence and burden of functional gastrointestinal disorders, results of Rome Foundation Global Study. Gastroenterology. 2020 Apr 12;S0016-5085(20)30487-X. doi: 10.1053/j.gastro.2020.04.014. Online ahead of print.

21. Gawron AJ, Bell R, Abu Dayyeh BK, Buckley FP, Chang K, Dunst CM, et al. Surgical and endoscopic management options for patients with GERD based on proton pump inhibitor symptom response: recommendations from an expert U.S. panel. Gastrointest Endosc. 2020;92:78-87. e2.

22. Halland M, Pandolfino J, Barba E. Diagnosis and treatment of rumination syndrome. Clin Gastroenterol Hepatol. 2018;16:1549-55.

23. Vachhani H, Ribeiro BS, Schey R. Rumination syndrome: Recognition and treatment. Curr Treat Options Gastroenterol. 2020 Jan 20. doi: 10.1007/ s11938-020-00272-4. Online ahead of print.

24. Absah I, Rishi A, Talley NJ, Katzka D, Halland M. Rumination syndrome: pathophysiology, diagnosis, and treatment. Neurogastroenterol Motil. 2017;29(4)

25. Call C, Walsh BT, Attia E. From DSM-IV to DSM-5: changes to eating disorder diagnoses. Curr Opin Psychiatry. 2013;26:532-6.

26. Hejazi RA, McCallum RW. Rumination syndrome: a review of current concepts and treatments. Am J Med Sci. 2014;348:324-9.

27. Sirota NA, Moskovchenko DV, Yaltonsky VM, Makarova IA, Yaltonskaya AV. [Cognitive therapy of depressive rumination]. Zh Nevrol Psikhiatr Im S S Korsakova. 2019;119:62-8.

28. Blondeau K, Boecxstaens V, Rommel N, Farré R, Depeyper S, Holvoet L, et al. Baclofen improves symptoms and reduces postprandial flow events in patients with rumination and supragastric belching. Clin Gastroenterol Hepatol. 2012;10:379-84.

29. Schmulson M. La neurogastroenterología en la práctica clínica. Neurogastro LATAM Rev. 2020;4:76-94.

30. Fox MR, Kahrilas PJ, Roman S, Gyawali CP, Scott SM, Rao SS, et al. Clinical measurement of gastrointestinal motility and function: who, when and which test? Nat Rev Gastroenterol Hepatol. 2018;15:568-79.

31. Schmulson M. Avances en neurogastroenterología. En: Madrazo de la Garza J, editor. Nutrición y gastroenterología pediátrica. 2. ${ }^{a}$ ed. McGraw Hill; 2015. pp. 241-245.

32. Sato H, Takahashi K, Mizuno KI, Hashimoto S, Kawata Y, Mizusawa T, et al. Overlap in disease concept of functional esophageal disorders and minor esophageal motility disorders. J Gastroenterol Hepatol. 2019;34:1940-5.

33. Sweis R, Heinrich H, Fox M; International Working Group for GI Motility and Function. Variation in esophageal physiology testing in clinical practice: Results from an international survey. Neurogastroenterol Motil. 2018;30(3).

34. Rezaie A, Buresi M, Lembo A, Lin H, McCallum R, Rao S, et al. Hydrogen and methane-based breath testing in gastrointestinal disorders: The North American Consensus. Am J Gastroenterol. 2017;112:775-84.

35. Drossman DA, Hasler WL. Rome IV-Functional GI Disorders: Disorders of gut-brain interaction. Gastroenterology. 2016;150:1257-61.

36. Keefer L, Drossman DA, Guthrie E, Simrén M, Tillisch K, Olden K, et al. Centrally mediated disorders of gastrointestinal pain. Gastroenterology. 2016 Feb 19:S0016-5085(16)00225-0. doi: 10.1053/j.gastro.2016.02.034. Online ahead of print. 
37. Drossman DA, Chang L, Chey WD, Kellow J, Tack J, Whitehead WE. Rome IV: Multidimensional clinical profile or functional gastrointestinal disorders. Raleigh, NC: The Rome Foundation; 2016.

38. Gyawali CP, Savarino E, Lazarescu A, Bor S, Patel A, Dickman R, et al. Curriculum for neurogastroenterology and motility training: A report from the joint ANMS-ESNM task force. Neurogastroenterol Motil. 2018;30:e13341.

39. Jáquez JL, Lascurain L, Montoya JR. Obesidad, disbiosis y trastornos gastrointestinales funcionales en edades pediátricas. Neurogastro LATAM Rev. 2020;4:110-25.

40. Saulnier DM, Riehle K, Mistretta TA, Diaz MA, Mandal D, Raza S, et al. Gastrointestinal microbiome signatures of pediatric patients with irritable bowel syndrome. Gastroenterology. 2011;141:1782-91.

41. Horvath A, Dziechciarz P, Szajewska H. Meta-analysis: Lactobacillus rhamnosus GG for abdominal pain-related functional gastrointestinal disorders in childhood. Aliment Pharmacol Ther. 2011;33:1302-10.
42. Zoppi G, Cinquetti M, Luciano A, Benini A, Muner A, Bertazzoni Minelli E. The intestinal ecosystem in chronic functional constipation. Acta Paediatr. 1998;87:836-41.

43. Bergstrom A, Skov TH, Bahl MI, Roager HM, Christensen LB, Ejlerskov KT, et al. Establishment of intestinal microbiota during early life: a longitudinal, explorative study of a large cohort of Danish infants. Appl Environ Microbiol. 2014;80:2889-900.

44. Million M, Lagier JC, Yahav D, Paul M. Gut bacterial microbiota and obesity. Clin Microbiol Infect. 2013;19:305-13.

45. Nadal I, Santacruz A, Marcos A, Warnberg J, Garagorri JM, Moreno LA, et al. Shifts in clostridia, bacteroides and immunoglobulin-coating fecal bacteria associated with weight loss in obese adolescents. Int J Obes (Lond). 2009;33:758-67. 